\title{
Mesomorphic Phase Behavior in Comb-like Polymer- Surfactant Complex
}

\author{
Isamu Akiba*, Hiroyasu Masunaga, Shouichi Murata, Kanako Sasaki
}

1 Department of Chemical Processes and Environments, The University of Kitakyushu, 1-1 Hibikino, Wakamatsu, Kitakyushu 808-0135, Japan; +81-(0)93-6953385; akiba@env.kitakyu-u.ac.jp

(Received: 12 April, 2006; published: 12 July, 2006)

\begin{abstract}
It was found that a comb-like complex consisting of poly[2-( $N, N$ dimethylamino)ethyl acrylate] (PDAEA) and p-dodecylbenzene sulfonic acid (DBSA) formed mesomorphically ordered lamellar phase at room temperature where PDAEA and DBSA are in molten state. The lamellar phase shows optical anisotropy, although the individual components are always isotropic. In addition it is found that the optical anisotropy of the PDAEA-DBSA complex completely disappears with accompanying order-disorder transition of the lamellar morphology with elevating temperature.
\end{abstract}

\section{Introduction}

A comb-like complex of polymer and amphiphilic molecule spontaneously forms mesoscopically ordered phase like a covalently connected comb-like polymer [1-6]. Formation of the mesomorphic phase in the comb-like complex is derived by attractive interactions between repeating units of a polymer and polar head groups of amphiphilic molecules, repulsive interactions between the polymer and hydrophobic tails of the amphiphiles, and aggregations of the individual components. Therefore, morphologies and regularity of the mesomorphic phase of the comb-like complex should be controlled by tuning the balance of these interactions. When strong segregation is combined with the preferential association between the polymer and amphiphilic molecule, well-organized mesophase should be formed in the comb-like complex. The formations of the highly ordered mesophases in the comb-like complexes are expected to induce additional properties, such as optical anisotropy. If we can prepare an optically anisotropic material from isotropic polymer and surfactant, they should be expected to be a novel class of anisotropic materials.

In this study, we examine the formation of the comb-like complex consisting of poly[2( $N, N$-dimethylamino)ethyl acrylate] (PDAEA) and $p$-dodecylbenzene sulfonic acid (DBSA). The PDAEA-DBSA pair is considered as a system in which preferential association by proton transfer between sulfonic group of DBSA and tertiary amino group of PDAEA, and successive segregation between the PDAEA and hydrophobic tails of DBSA [1, 4e, 7, 8]. Therefore, the PDAEA-DBSA complex is expected to form well-organized mesophase. Accordingly, it is also expected that the complexation of PDAEA and DBSA induce the additional properties which are not present in individual components. The objective of this paper is the analysis of nano-organized 
phase structure of the PDAEA-DBSA complex by small angle $\mathrm{X}$-ray scattering and searching its anisotropic property.

\section{Results and discussion}

Complex formation of the PDAEA-DBSA mixture is investigated by FTIR. Fig. 1 shows FTIR spectra in the region of $800-1300 \mathrm{~cm}^{-1}$ for the PDAEA, DBSA, and PDAEA-DBSA mixture. The DBSA shows $-\mathrm{SO}_{3} \mathrm{H}$ absorbance band at $900 \mathrm{~cm}^{-1}$, although the PDAEA does not show any absorbance peaks around $900 \mathrm{~cm}^{-1}$. The $\mathrm{SO}_{3} \mathrm{H}$ absorbance band at $900 \mathrm{~cm}^{-1}$ completely disappears in the PDAEA-DBSA mixture. In addition, the PDAEA-DBSA mixture shows an absorbance peak at 1220 $\mathrm{cm}^{-1}$. This absorbance peak is attributed to the $\mathrm{SO}_{3}^{-}$absorption. The PDAEA and DBSA do not show such absorbance peak at $1220 \mathrm{~cm}^{-1}$, although the DBSA show a broad absorbance attributed to $\mathrm{C}\left(\mathrm{CH}_{2}\right)_{\mathrm{n}}$ around $1220 \mathrm{~cm}^{-1}$. The disappearance of $\mathrm{SO}_{3} \mathrm{H}$ band and generation of the $\mathrm{SO}_{3}{ }^{-}$band in the FTIR spectrum indicate complete proton transfer from $-\mathrm{SO}_{3} \mathrm{H}$ of the DBSA to $-\mathrm{N}\left(\mathrm{CH}_{3}\right)_{2}$ of PDAEA in the PDAEA-DBSA mixture $[1 \mathrm{a}, 8,12]$. Therefore, the PDAEA forms comb-like complex with the DBSA through the noncovalent bond between the PDAEA and the DBSA as shown in Fig. 2. The PDAEA is immiscible with aliphatic compounds. Hence, strongly repulsive interaction between the alkyl chains of the DBSA and the PDAEA is expected. The strong repulsion in the comb-like PDAEA-DBSA complex should be a driving force to form mesoscopically organized phase.

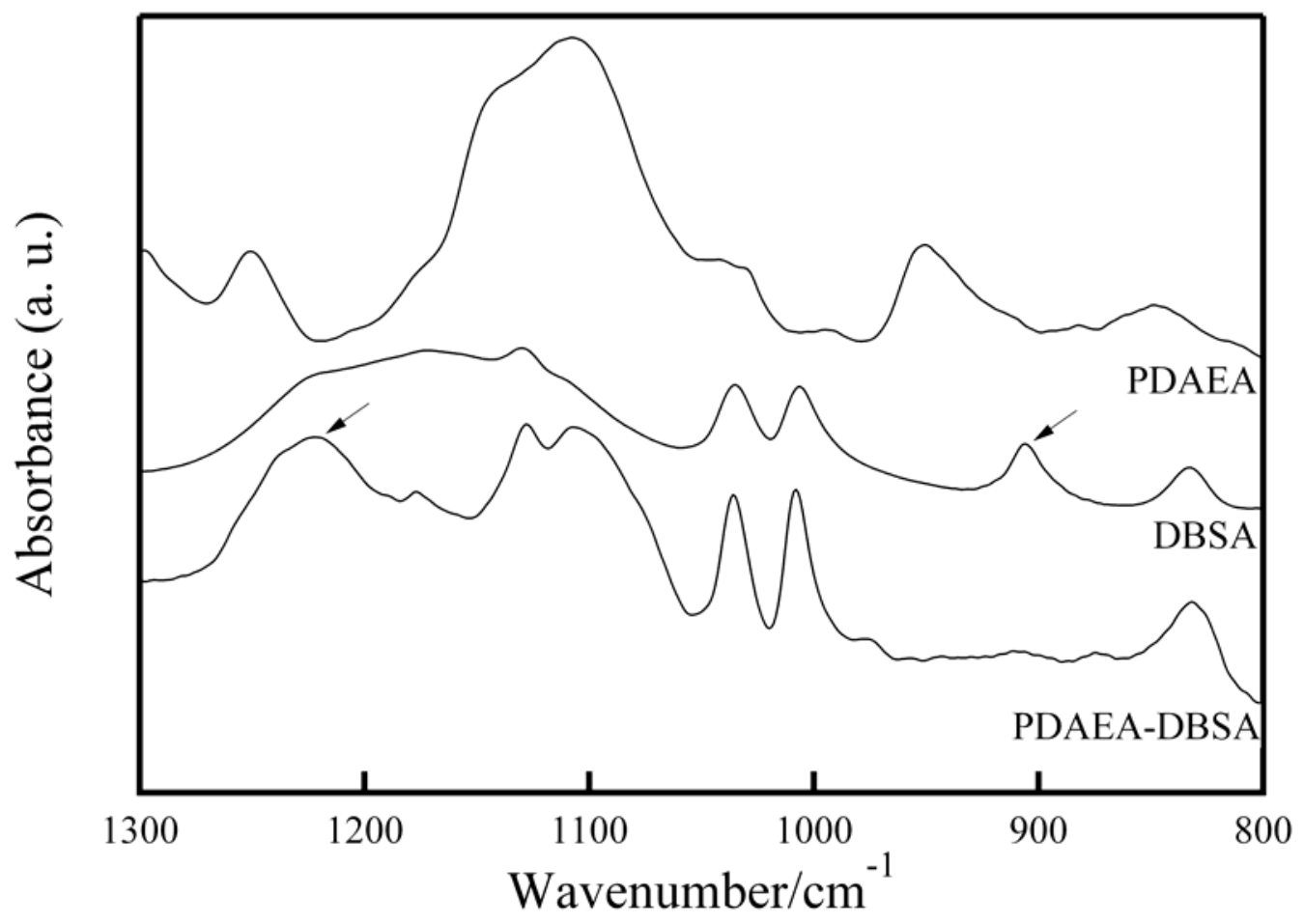

Fig. 1. FTIR spectra for PDAEA, DBSA, and PDAEA-DBSA complex.

At room temperature, the PDAEA, DBSA and PDAEA-DBSA complex are viscous fluids. In addition, the wide-angle $X$-ray diffraction measurements for them show diffuse halo, although data are not shown here. Therefore, they do not form crystalline order at room temperature. However, SAXS experiment indicates that the 
PDAEA-DBSA complex has ordered structure in mesoscopic scale. Fig. 3 shows one dimensional SAXS profiles of the PDAEA-DBSA complex, PDAEA, and DBSA at room temperature. In the SAXS profile of the PDAEA, any diffraction peaks are not recognized.

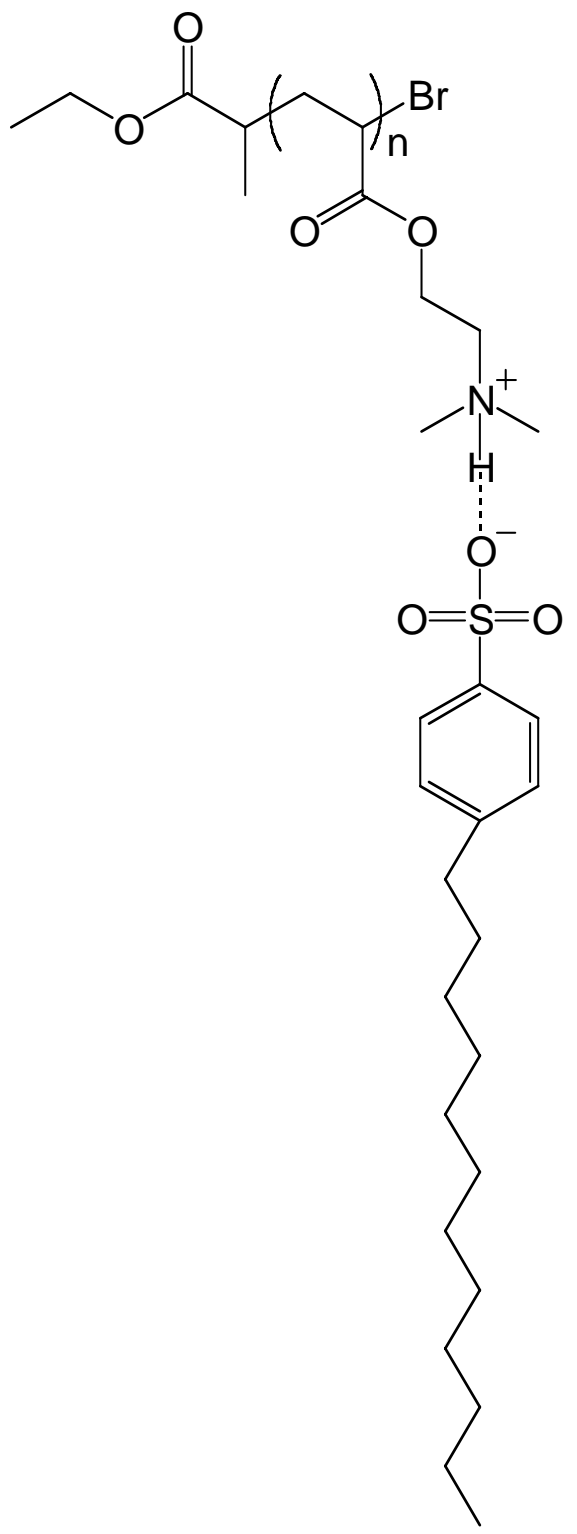

Fig. 2. Proton-transfer interaction between DBSA and PDAEA.

Therefore the PDAEA forms disordered liquid phase at room temperature. The SAXS profile of the DBSA shows broad peak at $2.9 \mathrm{~nm}^{-1}$. Since the DBSA is composed of a polar head and non-polar alkyl chain, the scattering peak of the DBSA should reflect the heterogeneity, such as a distorted lamellar with $2.2 \mathrm{~nm}$ period. On the contrary, the SAXS profile of the PDAEA-DBSA complex shows sharp diffraction peaks up to second order diffraction. The $q$ positions of the first- and second-order diffraction peaks are 2.06 and $4.12 \mathrm{~nm}^{-1}$, respectively. Because the $q$ positions of the diffraction peaks are relatively assigned to 1:2, the PDAEA-DBSA complex forms lamellar morphology with $3.0 \mathrm{~nm}$ of the periodic length. The first-order diffraction peak of the PDAEA-DBSA complex is extremely sharp, although it is a viscous liquid-like material at room temperature. In addition, the second-order diffraction peak is clearly 
observed. If the other ordered structures co-exist in this system, other higher order peaks relatively assigned to $3^{1 / 2}, 2^{1 / 2}$, and so on have to be observed. Hence, the lamellar structure of the PDAEA-DBSA complex is highly ordered. Because of the strong association between the $-\mathrm{SO}_{3} \mathrm{H}$ and $-\mathrm{N}\left(\mathrm{CH}_{3}\right)_{2}$ and strong repulsion between the alkyl tails of the DBSA and the PDAEA or the polar groups, the sharp interface should be formed in the lamellar of the PDAEA-DBSA complex. Consequently, the mesoscopic lamellar structure in the PDAEA-DBSA complex should be highly ordered. Because the periodic length of the lamellar structure of the PDAEA-DBSA complex is longer than that of the pure DBSA $(2.2 \mathrm{~nm})$, the lamellar structure of the PDAEA-DBSA complex consists of alternating the PDAEA and the DBSA layers.

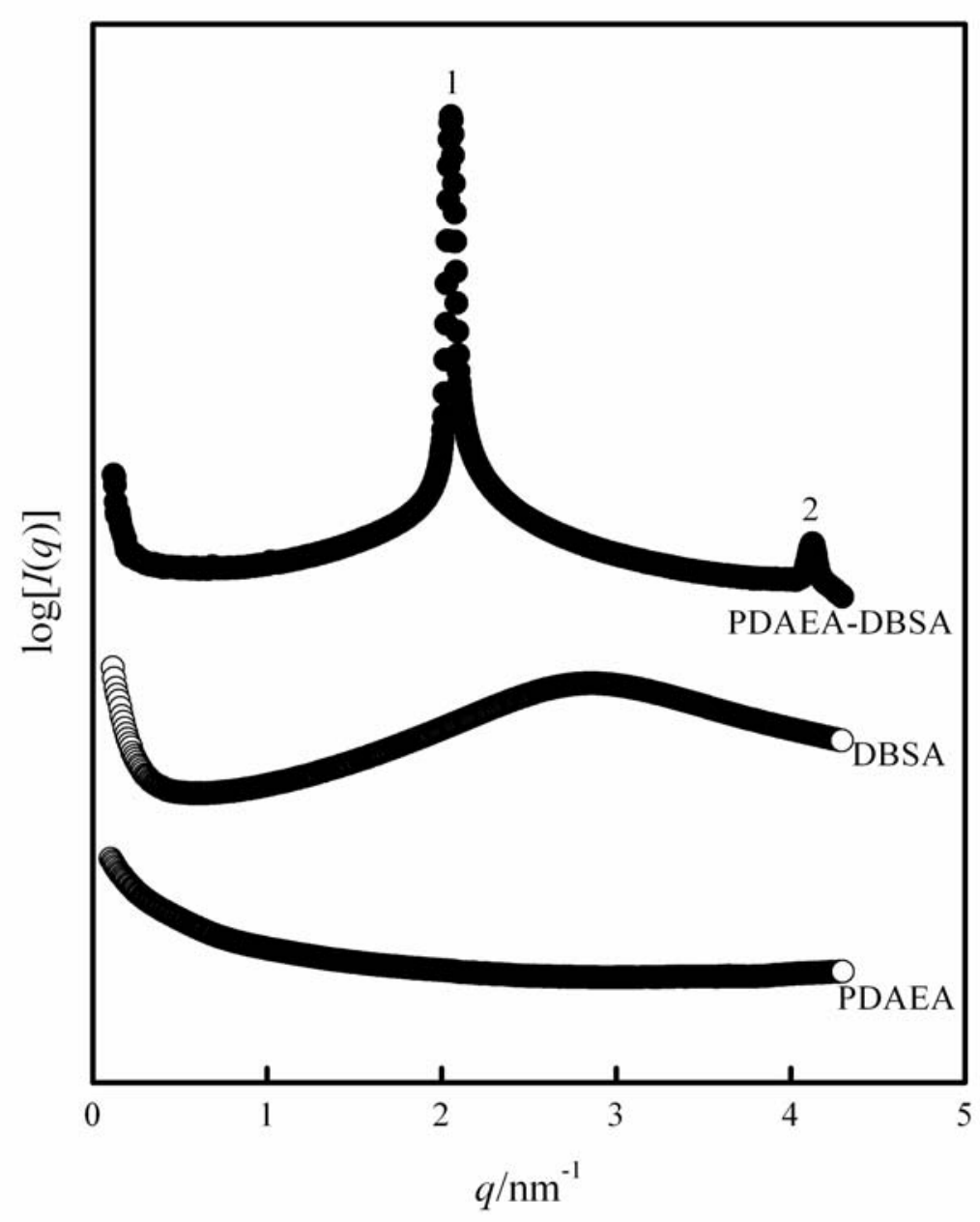

Fig. 3. SAXS profiles of PDAEA, DBSA, and PDAEA-DBSA complex.

The thicknesses of the individual layers are evaluated from one-dimensional correlation function $\gamma(r)[8,13]$. The $\gamma(r)$ is defined as [14]

$\gamma(r)=\frac{1}{Q} \int_{0}^{\infty} q^{2} I(q) \cos (q r) d q$

where $Q$ is the scattering invariant defined as

$Q=\int_{0}^{\infty} q^{2} I(q) d q$ 
Fig. 4 shows plot of one-dimensional correlation function of the PDAEA-DBSA complex. The $L$ and $I_{\mathrm{p}}$ represented in Fig. 4 corresponds to long period of the lamellar and thickness of the thinner layer, respectively. In this case, the thinner layer is considered to be the PDAEA phase $[4,8]$. The $L$ and $I_{p}$ of the PDAEA-DBSA complex are determined as $3.0 \mathrm{~nm}$ and $1.1 \mathrm{~nm}$, respectively. The $L$ value precisely corresponds to the long period evaluated from the $q$ value of the first-order diffraction peak in the SAXS profile. Therefore, the thickness of the DBSA layer $\left(I_{s}\right)$ in the complex is estimated to $1.9 \mathrm{~nm}$. The estimated $I_{\mathrm{S}}$ is slightly shorter than the periodic length of lamellar of the pure DBSA $(2.2 \mathrm{~nm})$. This result is caused because of alignment of the $-\mathrm{SO}_{3} \mathrm{H}$ groups of the DBSA at the sharp interface between the PDAEA and DBSA.

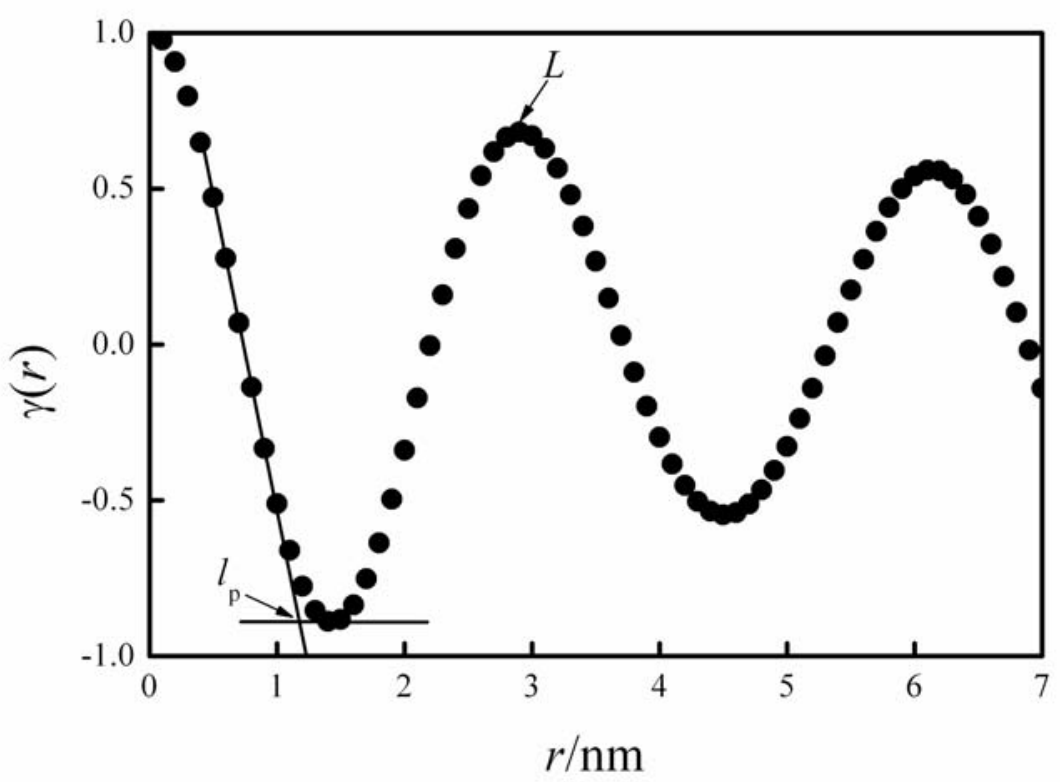

Fig. 4. One-dimensional correlation function of PDAEA-DBSA complex.

The mesoscopic organized structure of the PDAEA-DBSA complex is formed due to the balance of the noncovalent bond with the $-\mathrm{SO}_{3} \mathrm{H}$ and $-\mathrm{C}\left(\mathrm{CH}_{3}\right)_{2}$ and repulsive interaction between the PDAEA and DBSA. The balance of the interactions should be varied with varying temperature. If the dissociation between the $-\mathrm{SO}_{3} \mathrm{H}$ and $-\mathrm{N}\left(\mathrm{CH}_{3}\right)_{2}$ proceeds during the dissolution of alkyl chain of DBSA and PDAEA with elevating temperature, the complex should show macroscopic phase separation. However, the macroscopic phase separation is not observed for the PDAEA-DBSA complex with elevating temperature. On the contrary, the SAXS profile of the PDAEA-DBSA complex is drastically changed with elevating temperature. Fig. 5 shows SAXS profiles of the PDAEA-DBSA complex at room temperature and $90^{\circ} \mathrm{C}$. The scattering peaks observed in the SAXS profile of the PDAEA-DBSA complex at room temperature are entirely vanished at $90{ }^{\circ} \mathrm{C}$. This means that the lamellar structure of the PDAEA-DBSA complex collapses with elevating temperature. Therefore, the dissolution of the alkyl chains of the DBSA and PDAEA is preferentially occurring with elevating temperature. 


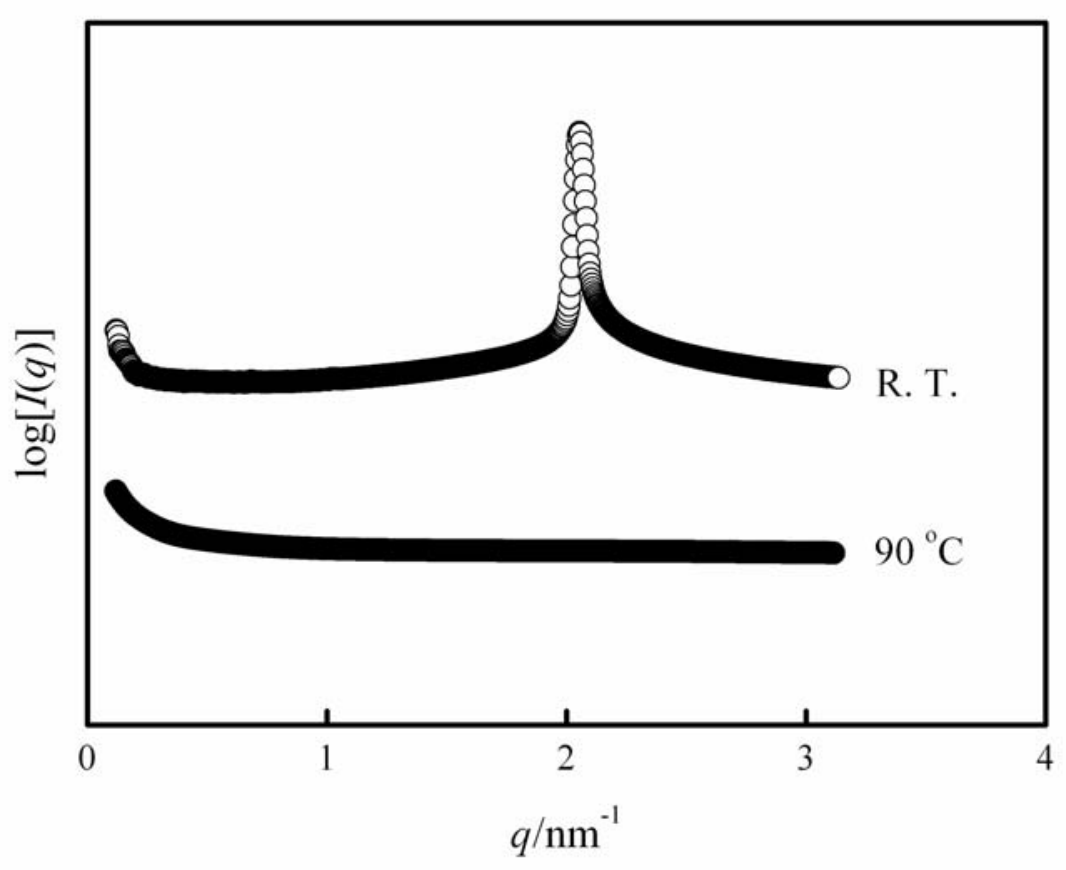

Fig. 5 Change in SAXS profile of PDAEA-DBSA complex with elevating temperature.

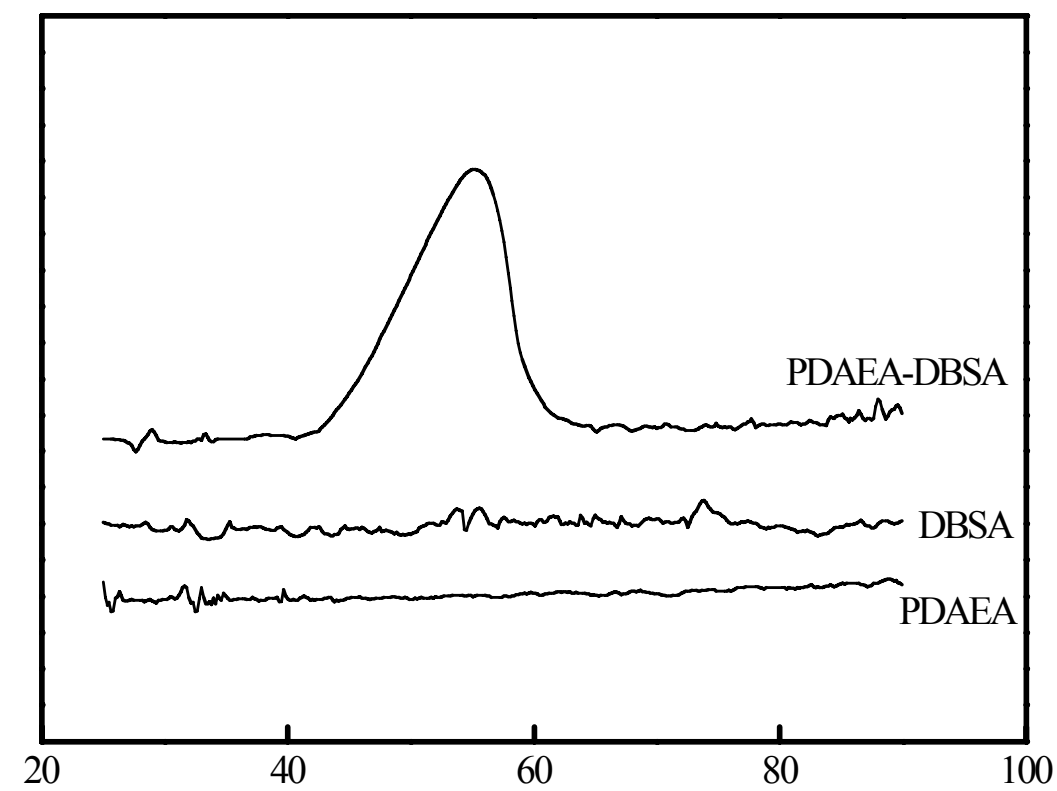

Fig. 6 DSC thermograms of PDAEA, DBSA, and PDAEA-DBSA complex.

In the region of the collapse of lamellar structure, thermal transition is also observed for the PDAEA-DBSA complex. Fig. 6 shows DSC thermograms of the PDAEA, DBSA, and the PDAEA-DBSA complex. The thermograms of the individual components do not show any transition peaks. On the other hand, the thermogram of the PDAEA-DBSA complex shows weak and broad endothermic peak with the onset 
at $45{ }^{\circ} \mathrm{C}$. Because the endothermic transition is characteristically observed for the PDAEA-DBSA complex, it should accompany with the order-disorder transition. However, the endothermic peak suggesting first-order thermodynamic phase transition, such as melting, is not generally observed in the order-disorder transition. Because the crystalline order is not recognized in the PDAEA-DBSA complex, the endothermic transition conceivably arise from liquid crystalline phase. Hence, the liquid crystalline properties, such as optical anisotropy, should be generated in the PDAEA-DBSA complex.

Fig. 7 shows polarized optical micrographs (POM) taken at room temperature and $90{ }^{\circ} \mathrm{C}$ for the PDAEA-DBSA complex. The POM photo at room temperature demonstrates optical anisotropy of the PDAEA-DBSA complex, although the individual components are optically isotropic. This result indicates that the liquid crystalline (LC) phase is generated by formation of the complex of the PDAEA with the DBSA. As mentioned above, the PDAEA-DBSA complex forms highly ordered lamellar structure. Therefore, the polar head groups of the DBSA are assembled at the interface between the PDAEA and DBSA phases. The optical anisotropy of the PDAEA-DBSA complex should be because of the spontaneous assembly of the DBSA molecules at the interface induced molecular orientation in local area. On the contrary, the optical anisotropy of the PDAEA-DBSA complex entirely disappears at $90{ }^{\circ} \mathrm{C}$. Therefore the PDAEA-DBSA complex undergoes phase transition from LC phase to isotropic liquid phase with elevating temperature. This result corresponds to the result of the SAXS and DSC measurements for the PDAEA-DBSA complex. Therefore, because of the order-disorder transition of the PDAEA-DBSA complex collapses the molecular orientation in local area, the LC-to-isotropic liquid phase transition of the complex should be occurring accompanied with the order-disorder transition.
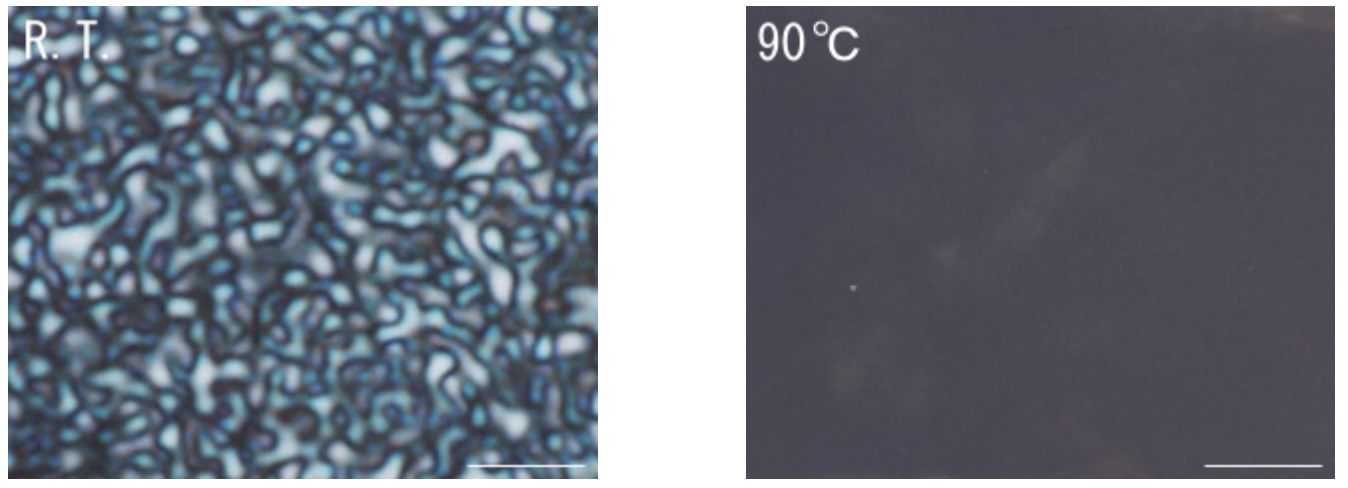

Fig. 7. Polarized optical micrographs of PDAEA-DBSA complex at room temperature and $90{ }^{\circ} \mathrm{C}$. Scale bars in these pictures indicate $10 \mu \mathrm{m}$.

\section{Experimental part}

$\mathrm{CuBr}$ was purchased from Nakalai Tesque Co., Ltd. The other reagents were purchased from Tokyo Chemical Industry Co., Ltd. The PDAEA was synthesized by Cu-mediated atom transfer radical polymerization (ATRP) technique as shown in Scheme 1 [11]. Ethyl 2-bromoisobutylate (I) as an initiator, 2-(N,N-Dimethyl amino)ethyl acrylate (M) as a monomer, and $N, N, N^{\prime}, N^{\prime \prime}, N$ "-pentamethyldiethylene triamine (L) as a ligand were added to dry flask with $\mathrm{CuBr}$. Here, molar ratio of $[\mathrm{M}] /[\mathrm{I}] /[\mathrm{L}] /[\mathrm{CuBr}]$ was set at 300/1/1/1. The solution was degassed with freeze-pump- 
thaw method for three times. The flask was sealed under vacuum and placed in an oil bath held at $80{ }^{\circ} \mathrm{C}$. After 1 hour, the polymerization was stopped by cooling and introducing air into the flask with vigorous stirring. The resulting product was dissolved in methanol and passed through a column packed with activated alumina for three times to remove copper. The methanol was removed in reduced pressure. The resulting PDAEA was further washed by $n$-hexane to remove residual monomer and dried in vacuum overnight. By gel permeation chromatography calibrated with standard polystyrenes, the $M_{n}$ and $M_{w}$ of the PDAEA were determined as $1.8 \times 10^{4} \mathrm{~g}$ $\mathrm{mol}^{-1}$ and $2.2 \times 10^{4} \mathrm{~g} \mathrm{~mol}^{-1}$, respectively. The PDAEA and DBSA are separately dissolved in methanol. The methanol solutions were mixed to be 1:1 molar ratio of the DBSA to repeating unit of PDAEA, followed by evaporation of the solvent. The resulting PDAEA-DBSA mixture is further dried in reduced pressure overnight.
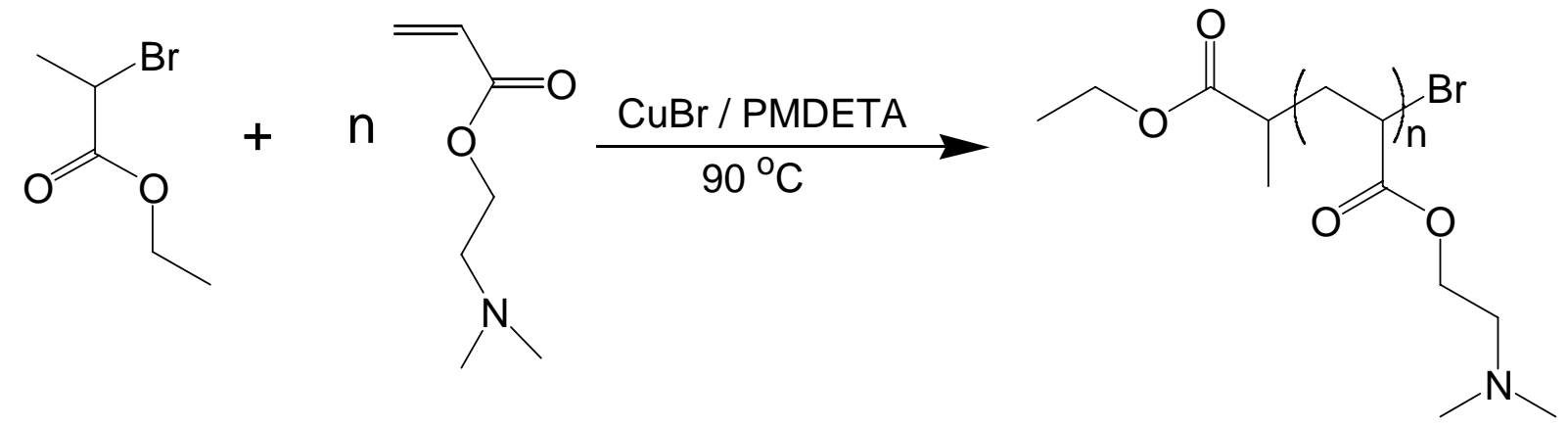

Scheme 1. Synthesis of PDAEA.

DSC thermograms were obtained by a Perkin Elmer Pyris 1 DSC. The DSC measurements were performed at a heating rate of 20 deg $\min ^{-1}$ under $\mathrm{N}_{2}$ atmosphere. FTIR spectra were obtained by a Perkin Elmer Spectrum GX FTIR spectrometer operating at $2 \mathrm{~cm}^{-1}$ resolution and 100 times integrations. Polarized optical micrographs were obtained using a Nikon Eclipse E-600 optical microscope. Small angle X-ray scattering (SAXS) measurements were carried out at the BL40B2 station at SPring-8, Japan. Two-dimensional (2-D) SAXS patterns were obtained by an imaging plate (Rigaku R-AXIS IV). One-dimensional SAXS profiles $(I(q)$ vs. $q)$ were obtained by conversion from 2-D SAXS patterns by circular averaging. Here, $I(q)$ is the scattering intensity and $q$ is the magnitude of scattering vector defined by following equation.

$q=\frac{4 \pi}{\lambda} \sin (\theta / 2)$

where $\lambda$ is wavelength of incident beam, and $\theta$ is scattering angle.

\section{Acknowledgements}

SAXS measurements were performed the SPring-8 with the approved number 2003A0297-NL2-np. FTIR and DSC measurements were carried out at Instrumentation Center of The University of Kitakyushu. This work is supported by funding from the MEXT via Kitakyushu Innovative Cluster Project. 


\section{References}

[1] (a) Ikkala, O.; Ruokolainen, J.; ten Brinke, G.; Torkkeli, M.; Serimaa, R. Macromolecules 1995, 28. (b) Ruokolainen, J.; ten Brinke, G.; Ikkala, O.; Torkkeli, M.; Serimaa, R. Macromolecules 1996, 29, 3409. (c) Ruokolainen, J.; Torkkeli, M.; Serimaa, R.; Komanschek, E.; ten Brinke, G.; Ikkala, O. Macromolecules 1997 30, 2002. (d) Vikki, T.; Ruokolainen, R.; Ikkala, O.; Passiniemi, P.; Isotalo, H.; Torkkeli, M.; Serimaa, R. Macromolecules 1997 30, 4064. (e) Ruokolainen, J.; Mäkinen, R.; Torkkeli, M.; Mäkelä, T.; Serimaa, R.; ten Brinke, G.; Ikkala, O. Science 1998, 280, 557. (f) Mäki-Ontto, R.; de Moel, K.; Polushkin, E.; van Ekenstein, G. A.; ten Brinke, G.; Ikkala, O. Adv. Mater. 2002, 14, 357.

[2] (a) Antonietti, M.; Conrad, J.; Thunemann, A. Macromolecules 1994, 27, 6007. (b) Antonietti, M.; Burger, C.; Effing, J. Adv. Mater. 1995, 7, 751.

[3] (a) Thünemann, A. F. Prog. Polym. Sci. , 2002, 27, 1473. (b) MacKnight, W. J.; Ponomarenko, E. A.; Tirrell, D. A. Acc. Chem. Res. 1998, 31, 781.

[4] (a) Akiba, I.; Akiyama, S. Macromolecules 1999, 32, 3741. (b) Akiba, I.; Akiyama, S. Macromolecules 2000, 33, 7967. (c) Akiba, I.; Akiyama, S. Mol. Cryst. Liq. Cryst. 2000, 339, 209. (d) Akiba, I.; Sakurai, K. e-Polym. 2002, 053. (e) Akiba, I.; Jeong, J.; Sakurai, K. Macromolecules 2003, 36, 8433. (f) Akiba, I.; Masunaga, H.; Sasaki, K.; Jeong, Y.; Sakurai, K.; Hara, S.; Yamamoto, K. Macromolecules 2004, 37, 1152.

[5] (a) Tanaka, F.; Ishida, M. Macromolecules 1997, 30, 1836. (b) Tanaka, F. Polym. J. 2002, 34, 479.

[6] (a) Ponomarenko, E. A.; Waddon, A. J.; Bakeev, K. N.; Tirrell, D. A.; MacKnight, W. J. Macromolecules 1996, 29, 4340. (b) Ponomarenko, E. A.; Tirrell, D. A.; MacKnight, W. J. Macromolecules 1996, 29, 8571. (c) Ponomarenko, E. A.; Tirrell, D. A.; MacKnight, W. J. Macromolecules 1998, 31, 1584.

[7] Tsiourvas, D.; Paleos, C. M.; Skoulios, A. Macromolecules 1999, 32, 8059.

[8] Chen, H.-L.; Hsiao, M. S. Macromolecules 1999, 32, 2967.

[9] (a) Kato, T.; Fréchet, J. M. J. Macromolecules 1989, 22, 3818. (b) Kato, T.; Fréchet, J. M. J. J. Am. Chem. Soc. 1989, 111, 8533. (c) Kato, T.; Fréchet, J. M. J. Macromolecules 1990, 23, 360. (d) Kato, T.; Fujishima, A.; Fréchet, J. M. J. Chem. Lett, 1990, 919. (e) Kumar, U.; Kato, T.; Fréchet, J. M. J. J. Am. Chem. Soc. 1992, 114, 6630. (f) Kato, T.; Kihara, H.; Uryu, T.; Fujishima, A.; Fréchet, J. M. J. Macromolecules 1992, 25 , 6836.

[10] (a) Ujiie, S.; limura, K. Chem. Lett, 1991, 411. (b) Ujiie, S.; limura, K. Macromolecules 1992, 25, 3174. (c) Bazuin, C. G.; Brandys, F. A. Chem. Mater. 1992, 4, 970. (d) Malik, S.; Dhal, P. K.; Mashelkar, R. A. Macromolecules 1995, 28, 2159.

[11] Zhang, X.; Matyjaszewski, K. Macromolecules 1999, 32, 1763.

[12] Akiba, I.; Masunaga, H.; Sasaki, K. Softmaterials. 2004, 2, 57.

[13] Strobl, G. R.; Schneider, M. J. Polym. Sci., Polym. Phys. Ed. 1980, 18, 1343.

[14] Vonk, C. G. J. Appl. Crystallogr. 1973, 6, 81. 